OPEN ACCESS

Edited by:

Consolato M. Sergi, Children's Hospital of Eastern Ontario

(CHEO), Canada

Reviewed by:

Min Gi,

Osaka City University, Japan Joseph Feulefack,

University of Alberta, Canada

${ }^{*}$ Correspondence:

Chen Xudong

913352729@qq.com

Shen Aiguo

850006516@qq.com

${ }^{+}$These authors have contributed equally to this work

Specialty section:

This article was submitted to Network Bioinformatics,

a section of the journal

Frontiers in Bioinformatics

Received: 26 June 2021 Accepted: 07 September 2021 Published: 12 October 2021

Citation:

Min Z, Xunlei Z, Haizhen C, Wenjing Z, Haiyan $Y$, Xiaoyun $L$, Jianyun $Z$, Xudong $C$ and Aiguo $S$ (2021) The Clinicopathologic and Prognostic Significance of c-Myc Expression in Hepatocellular Carcinoma: A Meta-

Analysis.

Front. Bioinform. 1:706835 doi: 10.3389/fbinf.2021.706835

\section{The Clinicopathologic and Prognostic Significance of c-Myc Expression in Hepatocellular Carcinoma: A Meta-Analysis}

\author{
Zhao Min ${ }^{1 \dagger}$, Zhang Xunlei ${ }^{2 \dagger}$, Chen Haizhen ${ }^{3+}$, Zhao Wenjing ${ }^{3}$, Yu Haiyan ${ }^{1}$, Lu Xiaoyun ${ }^{1}$, \\ Zhou Jianyun ${ }^{1}$, Chen Xudong ${ }^{1 *}$ and Shen Aiguo ${ }^{3 *}$ \\ ${ }^{1}$ Department of Pathology, Affiliated Tumor Hospital of Nantong University, Nantong, China, ${ }^{2}$ Department of Oncology, Affiliated \\ Tumor Hospital of Nantong University, Nantong, China, ${ }^{3}$ Cancer Research Center, Affiliated Tumor Hospital of Nantong \\ University, Nantong, China
}

Background: The incidence and mortality rates of hepatocellular carcinoma ( $\mathrm{HCC})$ are increasing worldwide. Therefore, there is an urgent need to elucidate the molecular drivers of HCC for potential early diagnosis and individualized treatment. Whether c-Myc expression plays a role in the clinicopathology and prognosis of patients with $\mathrm{HCC}$ remains controversial. This meta-analysis aimed to survey the prognostic role of c-Myc in HCC.

Methods: We searched PubMed, Cochrane Library, Embase, Web of Science, and Google Scholar databases for studies published through March 2020 that examined the association between c-Myc expression and clinicopathology or prognosis in HCC patients. The pooled hazard ratios (HRs) and 95\% confidence intervals (Cls) were used to investigate the prognostic significance of c-Myc expression. Odds ratios were calculated to evaluate the association between c-Myc expression and clinicopathologic features. We also tested for publication bias.

Results: Our meta-analysis included nine studies with 981 patients with HCC published between 1999 and 2016. A meta-analysis of these studies demonstrated that high c-Myc expression indicated a poor overall survival (OS) (HR $=2.260,95 \% \mathrm{Cl}: 1.660-3.080$, and $p<0.001)$ and disease-free survival (DFS) (HR $=1.770,95 \% \mathrm{Cl}: 1.430-2.450$, and $p<$ 0.001 ) in patients with HCC. However, high c-Myc expression was not associated with HBsAg, pathological type, TNM stage, or cirrhosis. We did not find any significant publication bias among the included studies, indicating that our estimates were robust and reliable.

Conclusion: c-Myc overexpression could predict poor OS and DFS in HCC patients. c-Myc could be a useful prognostic biomarker and therapeutic target for HCC.

Keywords: HCC, c-Myc, clinical pathology, prognostic, meta-analysis 


\section{INTRODUCTION}

Primary liver cancer (PLC) is a high-risk malignant tumor worldwide, with approximately one million new patients every year (Kulik and El-Serag, 2019). Hepatocellular carcinoma (HCC) is the main type of PLC, accounting for approximately $75-85 \%$ of cases (Bray et al., 2018). The incidence and mortality rates of HCC are increasing worldwide. Furthermore, most patients are diagnosed with middle- or advanced stage disease. Currently, there are no effective preventative or systemic treatments for HCC. Therefore, there is an urgent need to elucidate the molecular drivers of the initiation and progression of HCC for potential early diagnosis and individualized treatment.

c-Myc, a predominant member of the MYC family, is the most frequently activated oncogene. $\mathrm{c}-\mathrm{Myc}$ is a transcription factor that binds to the enhancer box sequence CACGTG (Caforio et al., 2018) and directly and indirectly regulates more than $15 \%$ of human genes involved in multiple biological processes, including cell proliferation, differentiation, tumorigenesis, apoptosis, and metabolism (Dang, 1999; Nesbit et al., 1999; Dang et al., 2006). $\mathrm{c}-\mathrm{Myc}$ gene amplification and overexpression are the main types of dysregulation in cancer. A variety of studies have reported an association between c-Myc dysregulation and the development of many malignancies, including HCC (Rapp et al., 2009; Ren et al., 2013; Fan et al., 2016). c-Myc is involved in the development of HCC, and almost $30 \%$ of human HCC samples show c-Myc gene amplification, which is correlated with protein expression; HBV, $\mathrm{HCV}$, and aflatoxin, the main risk factors of HCC, have also been reported to induce overexpression of c-Myc (Xu et al., 2019). However, the ability of c-Myc overexpression to predict patient prognosis remains controversial (Ji et al., 2018). In this study, we performed a systematic review to clarify the conflicting data regarding the association between c-Myc dysregulation and HCC prognosis.

\section{METHODS}

\section{Literature Search Strategy}

We comprehensively searched PubMed, Cochrane Library, Embase, Web of Science, and Google Scholar databases for studies published through March 2020. The following keywords were variably combined and used as the search criteria: hepatocellular cancer, HCC, liver cancer, c-Myc, and prognosis. Additional relevant references cited in the retrieved articles were also included in the evaluation.

\section{Eligibility Criteria}

The following criteria were used to identify articles eligible for inclusion in this systematic review: 1) published in English with the full text available; 2) all patients were diagnosed with HCC based on pathology; 3) c-Myc expression levels were detected in tumor tissues by fluorescence in situ hybridization, genome quantitative polymerase chain reaction, immunohistochemistry, or other techniques; and 4) the clinical characteristics of c-Myc dysregulation were compared with detailed survival information, including overall survival (OS) and disease-free survival (DFS).

\section{Data Extraction}

The available data were independently extracted by two reviewers (Zhao M and Zhang XL). Any dispute between the two reviewers was resolved by consensus or by a third reviewer (Chen HZ). The following details of each publication were extracted: the name of the first author; year of publication; country; ethnicity of the patients; number of patients; assessment method of c-Myc; cutoff value; follow-up time; clinicopathologic parameters, including sex, age, tumor size, tumor number, HBsAg, pathological type, cirrhosis, and TNM stage; OS or DFS; and outcomes of univariate and/or multivariate analyses [including hazard ratios (HRs), $p$-values, and $95 \%$ confidence intervals (CIs)]. Multivariate analysis results were selected when both univariate and multivariate analysis results were available in the same article.

\section{Quality Assessment}

Two investigators independently conducted quality assessments for all included studies (Zhao WY and Yu HY). The Newcastle-Ottawa Quality Assessment Scale, recommended by the Cochrane Collaboration as a risk-of-bias assessment tool for observational studies, was applied to assess the quality of the included studies (Stang, 2010; Shuster, 2011). Three parameters were judged for study quality: selection, comparability, and outcome assessment. Each study was scored from 0 to 9 according to these parameters. Any disputes were resolved through discussion.

\section{Statistical Analysis}

We used Cochrane's $\mathrm{Q}$ test and $p$-values to assess the heterogeneity among the eligible studies. A chi-squared $p$ value $<0.1$ or an $I^{2}$ value $>50 \%$ was defined as statistically significant heterogeneity (Cochran, 1950; Higgins et al., 2003). When heterogeneity existed [I (Bray et al., 2018) $\geq 50 \%$ ], a random-effects model was used; otherwise, a fixed-effects model was used. We used HRs with $95 \%$ CIs to measure the prognostic value of c-Myc in survival in HCC patients. An HR > 1 implied poorer survival for the higher c-Myc expression group. In contrast, an $\mathrm{HR}<1$ implied poorer survival for the lower c-Myc expression group. Odds ratios (ORs) and their associated 95\% CIs were used to assess the relationship between c-Myc overexpression and clinicopathologic features. An OR $>1$ represented poorer prognosis for the higher c-Myc expression group, whereas an $\mathrm{OR}<1$ indicated poorer prognosis for the lower c-Myc expression group. We extracted relevant data from the identified studies, and the ORs and HRs were estimated using a meta-analysis. All data analyses were performed using Revman 5.3 (RevMan, the Cochrane Collaboration) and Stata 11.0 (Stata, College Station). Sensitivity analysis was conducted to evaluate the stability of our estimates. Potential publication bias was evaluated using Begg's funnel plot and Egger's test (Egger et al., 1997). The $p$-values in the analysis were two-sided, and values less than 0.05 were regarded as statistically significant. 


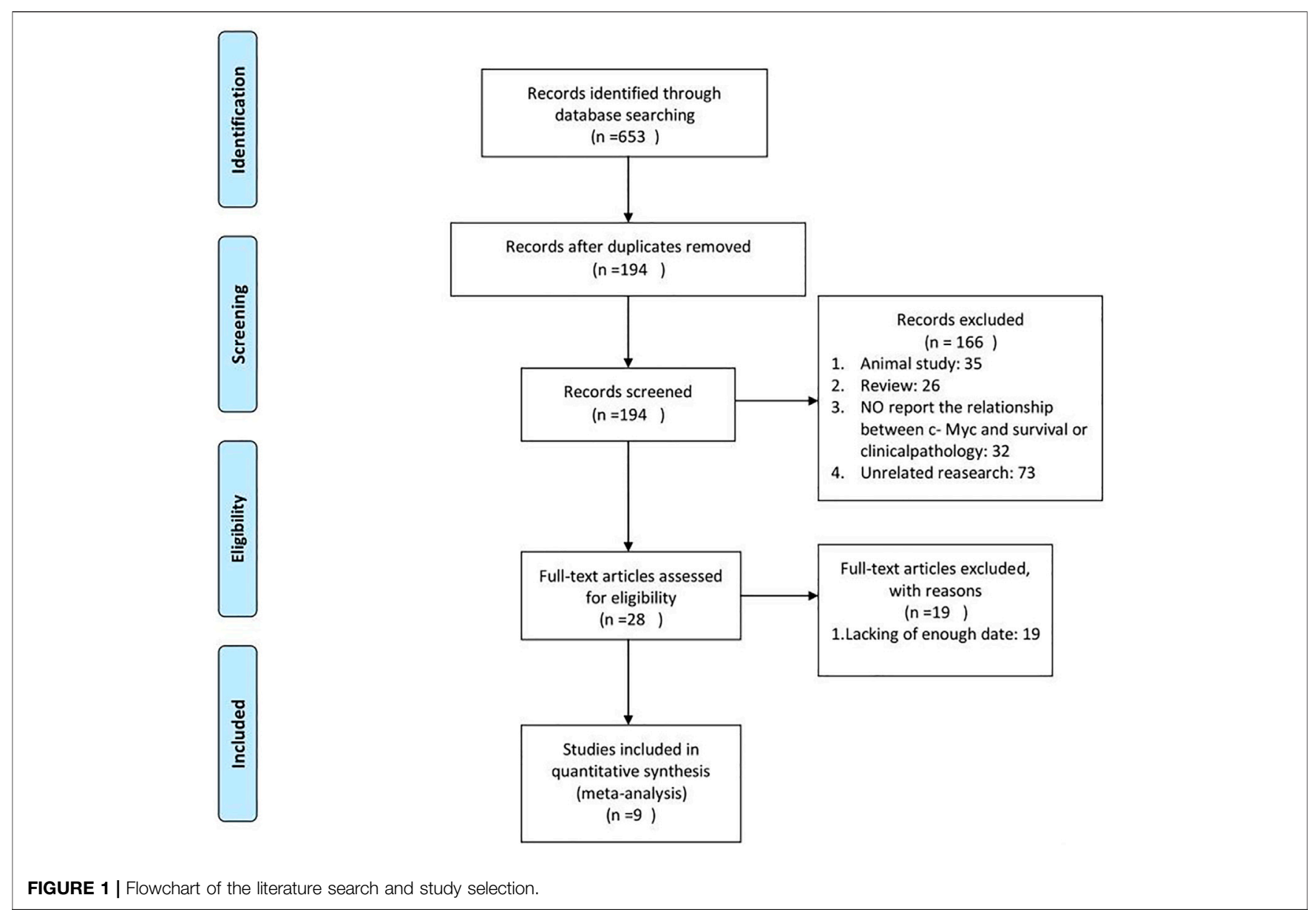

TABLE 1 | Characteristics of included studies.

\begin{tabular}{|c|c|c|c|c|c|c|}
\hline Ethnicity & Treatment & Follow-up (month) & Study period & Patients (n) & Survival analysis & NOS score \\
\hline Asian & Mixed & NA & $2007-2015$ & 37 & OS/DFS & 8 \\
\hline Asian & Surgery & NA & 2005-2007 & 88 & OS/DFS & 8 \\
\hline Asian & Surgery & NA & 1983-1992 & 40 & NA & 6 \\
\hline African & Surgery & NA & $2007-2013$ & 103 & NA & 6 \\
\hline Asian & Surgery & NA & 1991-1995 & 42 & DFS & 7 \\
\hline Asian & Surgery & 60 & 2007-2009 & 122 & OS/DFS & 8 \\
\hline American & Surgery & 432 & 1998-2009 & 154 & OS/DFS & 8 \\
\hline Asian & Surgery & NA & NA & 57 & NA & 6 \\
\hline Asian & Surgery & NA & NA & 54 & NA & 6 \\
\hline
\end{tabular}

\section{RESULTS}

\section{Search Results}

In this study, 653 articles were identified using the initial search strategy. Of these, 459 were removed because of duplication. After checking the titles and abstracts of the remaining articles, 28 full texts were further reviewed, and 9 studies were consistent with our purpose and included in the final analysis (Saegusa et al., 1993; Kawate et al., 1999; Liu et al., 2004; Jang et al., 2012; Wang et al., 2014; Huang et al., 2015; Abdou et al., 2016; Liu et al., 2016; Zheng et al., 2016). A flow diagram of the process is shown in Figure 1.

\section{Characteristics of the Included Studies}

The characteristics of the included studies are summarized in Table 1. The 9 studies were all retrospective cohort studies published between 1999 and 2016 and included 981 patients, whose data were used in the comprehensive analysis. Seven studies (77.8\%) included only Asian individuals, and two (22.2\%) included both Asian and American individuals. Among the nine studies, five reported a correlation between c-Myc expression and OS or DFS, and the remaining four showed a correlation between c-Myc expression and clinicopathologic features. 


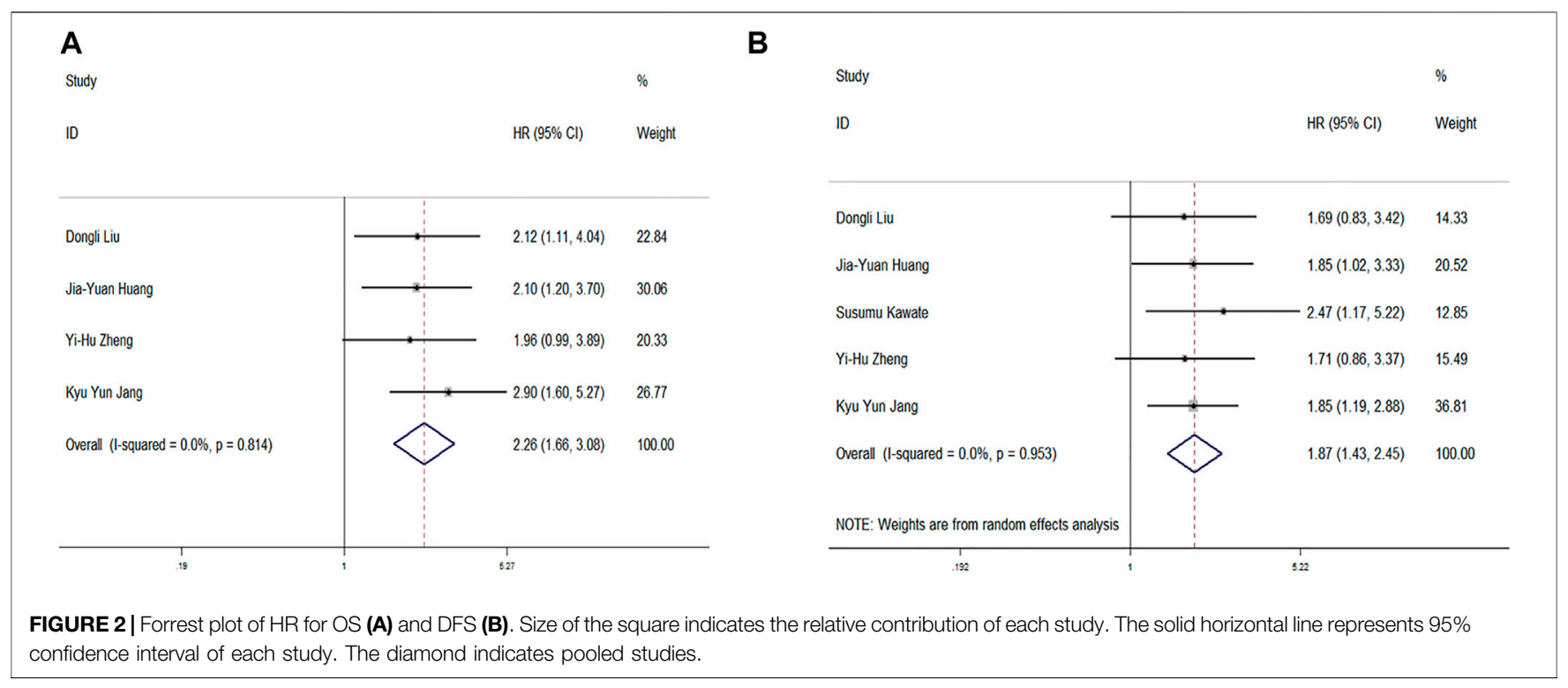

TABLE 2 | Relationship between high c-Myc and the clinicopathologic features.

\begin{tabular}{|c|c|c|c|c|c|c|c|}
\hline \multirow[t]{2}{*}{ Variable } & \multirow{2}{*}{$\begin{array}{l}\text { No. of } \\
\text { studies }\end{array}$} & \multirow{2}{*}{$\begin{array}{c}\text { No. of } \\
\text { patients }\end{array}$} & \multirow[t]{2}{*}{ Effects model } & \multirow{2}{*}{$\begin{array}{c}\text { OR } \\
(95 \% \mathrm{Cl})\end{array}$} & \multirow[t]{2}{*}{$\mathbf{P}$} & \multicolumn{2}{|c|}{ Heterogeneity } \\
\hline & & & & & & $I^{2}(\%)$ & $\mathbf{P}_{\mathbf{H}}$ \\
\hline BbsAg (positive vs. negative) & 3 & 383 & Random & $0.600(0.200-1.795)$ & 0.361 & 94.9 & $<0.001$ \\
\hline Pathological type (well + mode vs. poor) & 2 & 97 & Fixed & $0.867(0.649-1.156)$ & 0.331 & 0 & 1.424 \\
\hline TNM(I vs. II-III) & 2 & 96 & Random & $1.289(0.938-1.771)$ & 0.117 & 57 & 0.127 \\
\hline Cirrhosis (no vs. yes) & 3 & 197 & Fixed & $1.160(0.895-1.504)$ & 0.261 & 40.70 & 0.185 \\
\hline
\end{tabular}

\section{c-Myc Expression and Cancer Prognosis}

Four studies provided data on OS. High c-Myc expression was significantly associated with poor OS ( $\mathrm{HR}=2.260,95 \% \mathrm{CI}$ : 1.660-3.080, and $p<0.001$ ) (Figure 1). No statistically significant heterogeneity was observed $\left(p=0.814, I^{2}=82.1 \%\right)$. High c-Myc expression was also associated with poorer DFS in the random model ( $\mathrm{HR}=1.770,95 \% \mathrm{CI}: 1.430-2.450$, and $p<$ $0.001)$. However, high heterogeneity among the studies was observed ( $p<0.001$ and $I^{2}=95.30 \%$ ) (Figure 2B).

\section{c-Myc Expression and Clinical Features}

We next calculated the association between elevated c-Myc expression and clinical parameters (Table 2). Overexpression of c-Myc was not significantly associated with positive $\mathrm{HBsAg}$ $(\mathrm{OR}=0.600,95 \% \mathrm{CI}: 0.200-1.795$, and $p=0.361)$, pathological type (OR $=0.867,95 \% \mathrm{CI}: 0.649-1.156$, and $p=0.331)$, TNM stage $(\mathrm{OR}=1.289,95 \% \mathrm{CI}: 0.938-1.771$, and $p=0.117)$, or cirrhosis $(\mathrm{OR}=1.160,95 \%$ CI: 0.895-1.504, $p=0.261)$.

\section{Publication Bias and Sensitivity Analyses}

We used Begg's funnel plot and Egger's test to examine potential publication bias. Sensitivity analysis was executed by sequentially omitting each trial one at a time. As shown in Figure 3, there was no significant publication bias for OS and DFS analysis (Egger's test: $p=0.635$ for OS and $p=0.706$ for
DFS). The results of the sensitivity analysis demonstrated that our assessment was accurate and reliable. Due to the limited number of articles included, sensitivity analyses of the association between c-Myc expression and clinical features were not performed.

\section{DISCUSSION}

This meta-analysis analyzed the association between c-Myc expression and prognosis among patients with HCC. We showed that high $\mathrm{c}-\mathrm{Myc}$ expression was significantly associated with prognosis.

Cell survival and growth are promoted by c-Myc via the upregulation of downstream proteins involved in protein translation, cell cycle regulation, apoptosis, and metabolism (Chanvorachote et al., 2020). Cancer cell behaviors and activities, such as cell growth, survival, resistance to chemotherapies, immune surveillance, and metastasis, are also controlled by these effectors of c-Myc. c-Myc can regulate carcinogenesis and progression in many cancers, including lung cancer (Xiong et al., 2020), breast cancer (Chen et al., 2020), and colorectal cancer (Pan et al., 2020). c-Myc also plays an important role in the occurrence and development of HCC (Cheng et al., 2015; Attallah et al., 2017). 


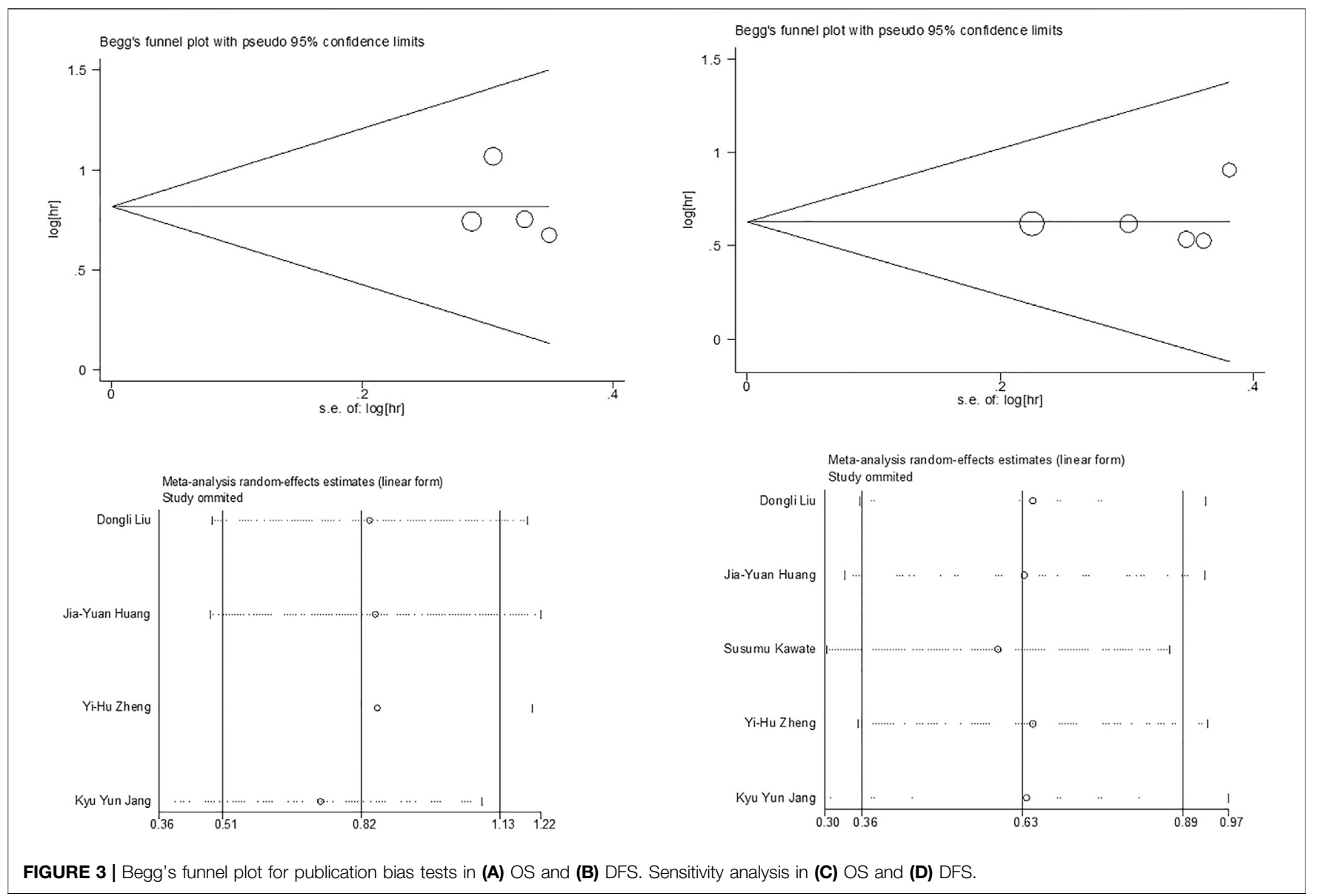

c-Myc is considered an important therapeutic target because of its dysregulation in approximately $50 \%$ of human cancers (Chen et al., 2018). Abnormal overexpression of c-Myc is related to poor prognosis in several cancers (Gustafson and Weiss, 2010), and c-Myc is overexpressed in approximately $70 \%$ of tumors. In malignant tumors, increased expression of c-Myc is associated with a malignant phenotype. Recent studies have shown that c-Myc is overexpressed in $50-100 \%$ of breast cancers (Locker et al., 1989) and 50-75\% of NSCLCs (Xu et al., 2011; Jiang et al., 2016). However, the prognostic value of c-Myc in patients with HCC remains controversial. One report indicated that low expression of c-Myc could predict poor outcomes in patients with HCC after resection (Ji et al., 2018). However, another study showed that overexpression of c-Myc is correlated with poor prognosis in HCC patients (Locker et al., 1989). The Human Protein Atlas indicates that MYC is not prognostic in liver cancer. The relationship between c-Myc expression and the prognosis of HCC patients remains controversial. Therefore, it is necessary to further identify the prognostic values of c-Myc expression in HCC. We performed this meta-analysis to clarify the association of c-Myc overexpression with survival and clinicopathologic features of HCC patients.

In this meta-analysis, we included nine articles including 981 patients with HCC to accurately assess this issue. Our results indicated that overexpression of $\mathrm{c}-\mathrm{Myc}$ significantly predicted poor OS and DFS in patients with HCC. This implies that c-Myc may be a biological marker of poor prognosis, and its expression can be used as a risk assessment tool for HCC patients. However, c-Myc expression was not associated with any clinicopathologic parameters we assessed, including HBsAg, pathological type, TNM stage, and cirrhosis. This may be because there were not enough patients included in our analysis. Additionally, a high degree of heterogeneity complicated the analysis. Our systematic review of a large number of researched studies shows that MYC has a known effect on the prognosis of liver cancer, although the Human Protein Atlas indicates that MYC is not prognostic in liver cancer. The reason may be that most of these studies included Asians, because of ethnic differences. Our research results indicated that c-Myc overexpression could predict poor OS and DFS in HCC. It is necessary to study the relationship between c-Myc expression and clinicopathologic characteristics in the future.

Although significant efforts have been made to conduct a comprehensive analysis, this meta-analysis has some limitations. First, all the studies in this meta-analysis were written in English. This might have caused publication bias to some extent, although we did not identify any publication bias. In the process of searching for references, we ruled out one animal study that was published in a language other than English. Therefore, there 
were also other reasons for exclusion. Second, our results might not apply to patients of other ethnicities because most of the patients in this study were from Asia. Third, there were not enough patients to conduct a good pooled analysis. Because of the lack of relevant data, we did not analyze all clinicopathologic features in this meta-analysis. Although our meta-analysis suggests that $\mathrm{c}-\mathrm{Myc}$ is associated with HCC prognosis because of these limitations, detailed clinical studies are warranted to establish the prognostic prediction value of $\mathrm{c}-\mathrm{Myc}$ in $\mathrm{HCC}$ in the future.

\section{CONCLUSION}

This meta-analysis revealed that c-Myc overexpression predicts poor OS and DFS in patients with HCC. Our results suggest that $\mathrm{c}-\mathrm{Myc}$ is a useful prognostic biomarker and therapeutic target for HCC.

\section{REFERENCE}

Abdou, A. G., Abd-Elwahed, M., Badr, M., Helmy, M., Soliman, E. A., and Maher, D. (2016). The Differential Immunohistochemical Expression of P53, C-Jun, C-Myc, and P21 between HCV-Related Hepatocellular Carcinoma with and without Cirrhosis. Appl. Immunohistochem. Mol. Morphol. : AIMM 24 (2), 75-87. doi:10.1097/PAI.0000000000000155

Attallah, A. M., El-Far, M., Abdelrazek, M. A., Omran, M. M., Attallah, A. A., Elkhouly, A. A., et al. (2017). Combined Use of Nuclear Phosphoprotein C-Myc and Cellular Phosphoprotein P53 for Hepatocellular Carcinoma Detection in High-Risk Chronic Hepatitis C Patients. Br. J. Biomed. Sci. 74 (4), 170-175. doi:10.1080/09674845.2017.1334739

Bray, F., Ferlay, J., Soerjomataram, I., Siegel, R. L., Torre, L. A., and Jemal, A. (2018). Global Cancer Statistics 2018: GLOBOCAN Estimates of Incidence and Mortality Worldwide for 36 Cancers in 185 Countries. CA: a Cancer J. clinicians 68 (6), 394-424. doi:10.3322/caac.21492

Caforio, M., Sorino, C., Iacovelli, S., Fanciulli, M., Locatelli, F., and Folgiero, V. (2018). Recent Advances in Searching C-Myc Transcriptional Cofactors during Tumorigenesis. J. Exp. Clin. Cancer Res. : CR 37 (1), 239. doi:10.1186/s13046018-0912-2

Chanvorachote, P., Sriratanasak, N., and Nonpanya, N. (2020). C-myc Contributes to Malignancy of Lung Cancer: A Potential Anticancer Drug Target. Anticancer Res. 40 (2), 609-618. doi:10.21873/anticanres.13990

Chen, H., Liu, H., and Qing, G. (2018). Targeting Oncogenic Myc as a Strategy for Cancer Treatment. Signal. Transduction Targeted Therapy 3, 5. doi:10.1038/ s41392-018-0008-7

Chen, R., Guo, S., Yang, C., Sun, L., Zong, B., Li, K., et al. (2020). Although cMYC Contributes to Tamoxifen Resistance, it Improves Cisplatin Sensitivity in ERpositive Breast Cancer. Int. J. Oncol. 56 (4), 932-944. doi:10.3892/ ijo.2020.4987

Cheng, Q., Yuan, F., Lu, F., Zhang, B., Chen, T., Chen, X., et al. (2015). CSIG Promotes Hepatocellular Carcinoma Proliferation by Activating C-MYC Expression. Oncotarget 6 (7), 4733-4744. doi:10.18632/oncotarget.2900

Cochran, W. G. (1950). The Comparison of Percentages in Matched Samples. Biometrika 37(3-4), 256-266.

Dang, C. V. (1999). c-Myc Target Genes Involved in Cell Growth, Apoptosis, and Metabolism. Mol. Cell. Biol. 19 (1), 1-11. doi:10.1128/mcb.19.1.1

Dang, C. V., O’Donnell, K. A., Zeller, K. I., Nguyen, T., Osthus, R. C., and Li, F. (2006). The C-Myc Target Gene Network. Semin. Cancer Biol. 16 (4), 253-264. doi:10.1016/j.semcancer.2006.07.014

Egger, M., Davey Smith, G., and Schneider, M. (1997). Minder C Bias in MetaAnalysis Detected by a Simple, Graphical Test. BMJ 315 (7109), 629-634. doi:10.1136/bmj.315.7109.629

\section{AUTHOR CONTRIBUTIONS}

ZM conceived and designed the protocol and study and drafted the manuscript. ZX and CH identified studies to be screened. ZW and YH identified studies for eligibility, extracted data, and assessed the methodological quality of included studies. LX and ZJ performed the analysis. CX and SA revised the manuscript. All the authors read and approved the final manuscript.

\section{FUNDING}

Our work was supported by a General Project of Nantong Health Committee (MA2020009), Jiangsu Province "333 Project" scientific research funding project (YYRC1701), Nantong Science and technology project (MS22019010), and Nantong health committee research projects (MB2020016).

Fan, L., Peng, G., Sahgal, N., Fazli, L., Gleave, M., Zhang, Y., et al. (2016). Regulation of C-Myc Expression by the Histone Demethylase JMJD1A Is Essential for Prostate Cancer Cell Growth and Survival. Oncogene 35 (19), 2441-2452. doi:10.1038/onc.2015.309

Gustafson, W. C., and Weiss, W. A. (2010). Myc Proteins as Therapeutic Targets. Oncogene 29 (9), 1249-1259. doi:10.1038/onc.2009.512

Higgins, J. P., Thompson, S. G., Deeks, J. J., and Altman, D. G. (2003). Measuring Inconsistency in Meta-Analyses. BMJ 327 (7414), 557-560. doi:10.1136/ bmj.327.7414.557

Huang, J. Y., Zhang, K., Chen, D. Q., Chen, J., Feng, B., Song, H., et al. (2015). MicroRNA-451: Epithelial-Mesenchymal Transition Inhibitor and Prognostic Biomarker of Hepatocelluar Carcinoma. Oncotarget 6 (21), 18613-18630. doi:10.18632/oncotarget.4317

Jang, K. Y., Noh, S. J., Lehwald, N., Tao, G. Z., Bellovin, D. I., Park, H. S., et al. (2012). SIRT1 and C-Myc Promote Liver Tumor Cell Survival and Predict Poor Survival of Human Hepatocellular Carcinomas. PloS one 7 (9), e45119. doi:10.1371/journal.pone.0045119

Ji, F., Zhang, Z. H., Zhang, Y., Shen, S. L., Cao, Q. H., Zhang, L. J., et al. (2018). Low Expression of C-Myc Protein Predicts Poor Outcomes in Patients with Hepatocellular Carcinoma after Resection. BMC cancer 18 (1), 460. doi:10.1186/s12885-018-4379-5

Jiang, R., Wang, X., Jin, Z., and Li, K. (2016). Association of Nuclear PIM1 Expression with Lymph Node Metastasis and Poor Prognosis in Patients with Lung Adenocarcinoma and Squamous Cell Carcinoma. J. Cancer 7 (3), 324-334. doi:10.7150/jca.13422

Kawate, S., Fukusato, T., Ohwada, S., Watanuki, A., and Morishita, Y. (1999). Amplification of C-Myc in Hepatocellular Carcinoma: Correlation with Clinicopathologic Features, Proliferative Activity and P53 Overexpression. Oncology 57 (2), 157-163. doi:10.1159/000012024

Kulik, L., and El-Serag, H. B. (2019). Epidemiology and Management of Hepatocellular Carcinoma. Gastroenterology 156 (2), 477-491 el. doi:10.1053/j.gastro.2018.08.065

Liu, D., Dong, L., Liu, Y., Wen, D., Gao, D., Sun, H., et al. (2016). A C-Myc/miR-17$5 p$ Feedback Loop Regulates Metastasis and Invasion of Hepatocellular Carcinoma. Tumour Biol. : J. Int. Soc. Oncodevelopmental Biol. Med. 37 (4), 5039-5047. doi:10.1007/s13277-015-4355-5

Liu, Y. C., Chen, C. J., Wu, H. S., Chan, D. C., Yu, J. C., Yang, A. H., et al. (2004). Telomerase and C-Myc Expression in Hepatocellular Carcinomas. Eur. J. Surg. Oncol. : J. Eur. Soc. Surg. Oncol. Br. Assoc. Surg. Oncol. 30 (4), 384-390. doi:10.1016/j.ejso.2004.01.003

Locker, A. P., Dowle, C. S., Ellis, I. O., Elston, C. W., Blamey, R. W., Sikora, K., et al. (1989). c-Myc Oncogene Product Expression and Prognosis in Operable Breast Cancer. Br. J. Cancer 60 (5), 669-672. doi:10.1038/ bjc. 1989.337 
Nesbit, C. E., Tersak, J. M., and Prochownik, E. V. (1999). MYC Oncogenes and Human Neoplastic Disease. Oncogene 18 (19), 3004-3016. doi:10.1038/ sj.onc. 1202746

Pan, W., Wang, W., Huang, J., Lu, K., Huang, S., Jiang, D., et al. (2020). The Prognostic Role of C-MYC Amplification in Schistosomiasis-Associated Colorectal Cancer. Jpn. J. Clin. Oncol. 50 (4), 446-455. doi:10.1093/jjco/ hyz210

Rapp, U. R., Korn, C., Ceteci, F., Karreman, C., Luetkenhaus, K., Serafin, V., et al. (2009). MYC Is a Metastasis Gene for Non-small-cell Lung Cancer. PloS one 4 (6), e6029. doi:10.1371/journal.pone.0006029

Ren, J., Jin, F., Yu, Z., Zhao, L., Wang, L., Bai, X., et al. (2013). MYC Overexpression and Poor Prognosis in Sporadic Breast Cancer with BRCA1 Deficiency. Tumour Biol. : J. Int. Soc. Oncodevelopmental Biol. Med. 34 (6), 3945-3958. doi:10.1007/ s13277-013-0983-9

Saegusa, M., Takano, Y., Kishimoto, H., Wakabayashi, G., Nohga, K., and Okudaira, M. (1993). Comparative Analysis of P53 and C-Myc Expression and Cell Proliferation in Human Hepatocellular Carcinomas-Aan Enhanced Immunohistochemical Approach. J. Cancer Res. Clin. Oncol. 119 (12), 737-744. doi:10.1007/BF01195346

Shuster, J. J. (2011). Review: Cochrane Handbook for Systematic Reviews for Interventions, Version 5.1.0, Published 3/2011, Julian P.T. Higgins and Sally Green, Editors Res. Synth. Methods 22, 126-130. doi:10.1002/jrsm.38

Stang, A. (2010). Critical Evaluation of the Newcastle-Ottawa Scale for the Assessment of the Quality of Nonrandomized Studies in Meta-Analyses. Eur. J. Epidemiol. 25 (9), 603-605. doi:10.1007/s10654-010-9491-Z

Wang, L., Zhang, X., Jia, L. T., Hu, S. J., Zhao, J., Yang, J. D., et al. (2014). c-Mycmediated Epigenetic Silencing of MicroRNA-101 Contributes to Dysregulation of Multiple Pathways in Hepatocellular Carcinoma. Hepatology 59 (5), 1850-1863. doi:10.1002/hep.26720

Xiong, J., Xing, S., Dong, Z., Niu, L., Xu, Q., Liu, P., et al. (2020). STK31 Regulates the Proliferation and Cell Cycle of Lung Cancer Cells via the Wnt/betacatenin
Pathway and Feedback Regulation by Cmyc. Oncol. Rep. 43 (2), 395-404. doi:10.3892/or.2019.7441

Xu, X., Sun, P. L., Li, J. Z., Jheon, S., Lee, C. T., and Chung, J. H. (2011). Aberrant Wnt1/beta-Catenin Expression Is an Independent Poor Prognostic Marker of Non-small Cell Lung Cancer after Surgery. J. Thorac. Oncol. : official Publ. Int. Assoc. Study Lung Cancer 6 (4), 716-724. doi:10.1097/JTO.0b013e31820c5189

Xu, Z., Xu, M., Liu, P., Zhang, S., Shang, R., Qiao, Y., et al. (2019). The mTORC2Aktl Cascade Is Crucial for C-Myc to Promote Hepatocarcinogenesis in Mice and Humans. Hepatology 70 (5), 1600-1613. doi:10.1002/hep.30697

Zheng, Y. H., Hu, W. J., Chen, B. C., Grahn, T. H., Zhao, Y. R., Bao, H. L., et al. (2016). BCAT1, a Key Prognostic Predictor of Hepatocellular Carcinoma, Promotes Cell Proliferation and Induces Chemoresistance to Cisplatin. Liver Int. : official J. Int. Assoc. Study Liver 36 (12), 1836-1847. doi:10.1111/liv.13178

Conflict of Interest: The authors declare that the research was conducted in the absence of any commercial or financial relationships that could be construed as a potential conflict of interest.

Publisher's Note: All claims expressed in this article are solely those of the authors and do not necessarily represent those of their affiliated organizations, or those of the publisher, the editors, and the reviewers. Any product that may be evaluated in this article, or claim that may be made by its manufacturer, is not guaranteed or endorsed by the publisher.

Copyright (C) 2021 Min, Xunlei, Haizhen, Wenjing, Haiyan, Xiaoyun, Jianyun, Xudong and Aiguo. This is an open-access article distributed under the terms of the Creative Commons Attribution License (CC BY). The use, distribution or reproduction in other forums is permitted, provided the original author(s) and the copyright owner(s) are credited and that the original publication in this journal is cited, in accordance with accepted academic practice. No use, distribution or reproduction is permitted which does not comply with these terms. 